\title{
The Effects of Thermal Radiation, Hall Currents, Soret, and Dufour on MHD Flow by Mixed Convection over a Vertical Surface in Porous Media
}

\author{
Stanford Shateyi, ${ }^{1}$ Sandile Sydney Motsa, ${ }^{2}$ and Precious Sibanda ${ }^{3}$ \\ ${ }^{1}$ Department of Mathematics and Applied Mathematics, University of Venda, Private Bag X5050, \\ Thohoyandou 0950, South Africa \\ ${ }^{2}$ Mathematics Department, University of Swaziland, Private Bag 4, M201 Kwaluseni, Swaziland \\ ${ }^{3}$ Department of Mathematics and Applied Mathematics, University of Natal, Private Bag X01, Scottsville, \\ Pietermaritzburg 3209, South Africa
}

Correspondence should be addressed to Stanford Shateyi, stanford.shateyi@univen.ac.za

Received 29 July 2009; Revised 14 January 2010; Accepted 8 March 2010

Academic Editor: Dane Quinn

Copyright (C) 2010 Stanford Shateyi et al. This is an open access article distributed under the Creative Commons Attribution License, which permits unrestricted use, distribution, and reproduction in any medium, provided the original work is properly cited.

\begin{abstract}
The study sought to investigate the influence of a magnetic field on heat and mass transfer by mixed convection from vertical surfaces in the presence of Hall, radiation, Soret (thermaldiffusion), and Dufour (diffusion-thermo) effects. The similarity solutions were obtained using suitable transformations. The similarity ordinary differential equations were then solved by MATLAB routine bvp4c. The numerical results for some special cases were compared with the exact solution and those obtained by Elgazery (2009) and were found to be in good agreement. A parametric study illustrating the influence of the magnetic strength, Hall current, Dufour, and Soret, Eckert number, thermal radiation, and permeability parameter on the velocity, temperature, and concentration was investigated.
\end{abstract}

\section{Introduction}

The range of free convective flows that occur in nature and in engineering practice is very large and has been extensively considered by many researchers (see, [1, 2], among others). When heat and mass transfer occur simultaneously between the fluxes, the driving potentials are of more intricate nature. An energy flux can be generated not only by temperature gradients but by composition gradients. The energy flux caused by a composition is called Dufour or diffusion-thermo effect. Temperature gradients can also create mass fluxes, and this is the Soret or thermal-diffusion effect. Generally, the thermal-diffusion and the diffusionthermo effects are of smaller-order magnitude than the effects prescribed by Fourier's or 
Fick's laws and are often neglected in heat and mass transfer processes. However, there are exceptions. The thermal-diffusion effect, for instance, has been utilized for isotope separation and in mixture between gases with very light molecular weight (Hydrogen-Hellium) and of medium molecular weight (Nitrogen-air) the diffusion-thermo effect was found to be of a magnitude such that it cannot be neglected (see Kafoussias and Williams [3] and references therein). Kafoussias and Williams [3] considered the boundary layer-flows in the presence of Soret, and Dufour effects associated with the thermal diffusion and diffusion-thermo for the mixed forced natural convection.

In recent years, progress has been considerably made in the study of heat and mass transfer in magneto hydrodynamic flows due to its application in many devices, like the MHD power generator and Hall accelerator. The influence of magnetic field on the flow of an electrically conducting viscous fluid with mass transfer and radiation absorption is also useful in planetary atmosphere research. Kinyanjui et al. [4] presented simultaneous heat and mass transfer in unsteady free convection flow with radiation absorption past an impulsively started infinite vertical porous plate subjected to a strong magnetic field. Yih [5] numerically analyzed the effect of transpiration velocity on the heat and mass transfer characteristics of mixed convection about a permeable vertical plate embedded in a saturated porous medium under the coupled effects of thermal and mass diffusion. Elbashbeshy [6] studied the effect of surface mass flux on mixed convection along a vertical plate embedded in porous medium.

Chin et al. [7] obtained numerical results for the steady mixed convection boundary layer flow over a vertical impermeable surface embedded in a porous medium when the viscosity of the fluid varies inversely as a linear function of the temperature. Pal and Talukdar [8] analyzed the combined effect of mixed convection with thermal radiation and chemical reaction on MHD flow of viscous and electrically conducting fluid past a vertical permeable surface embedded in a porous medium is analyzed. Mukhopadhyay [9] performed an analysis to investigate the effects of thermal radiation on unsteady mixed convection flow and heat transfer over a porous stretching surface in porous medium. Hayat et al. [10] analyzed a mathematical model in order to study the heat and mass transfer characteristics in mixed convection boundary layer flow about a linearly stretching vertical surface in a porous medium filled with a viscoelastic fluid, by taking into account the diffusionthermo (Dufour) and thermal-diffusion (Soret) effects.

Postelnicu [11] studied simultaneous heat and mass transfer by natural convection from a vertical plate embedded in electrically conducting fluid saturated porous medium, using Darcy-Boussinesq model including Soret, and Dufour effects. Lyubimova et al. [12] dealt with the numerical investigation of the influence of static and vibrational acceleration on the measurement of diffusion and Soret coefficients in binary mixtures, in low gravity conditions. Abreu et al. [13] examined the boundary layer solutions for the cases of forced, natural, and mixed convection under a continuous set of similarity type variables determined by a combination of pertinent variables measuring the relative importance of buoyant force term in the momentum equation.

Alam et al. [14] studied numerically the Dufour and Soret effects on combined free-forced convection and mass transfer flow past a semi-infinite vertical plate, under the influence of transversely applied magnetic field. Alam and Rahman [15] studied numerically the Dufour and Soret effects on mixed convection flow past a vertical plate embedded in a porous medium. Li et al. [16] took an account of the thermal-diffusion and diffusionthermo effects, to study the properties of the heat and mass transfer in a strongly endothermic chemical reaction system for a porous medium. 
Gaikwad et al. [17] investigated the onset of double diffusive convection in a twocomponent couple of stress fluid layer with Soret, and Dufour effects using both linear and nonlinear stability analysis.

The interaction of buoyancy with thermal radiation has increased greatly during the last decade due to its importance in many practical applications. The thermal radiation effect is important under many isothermal and nonisothermal situations. If the entire system involving the polymer extrusion process is placed in a thermally controlled environment, then thermal radiation could be important. The knowledge of radiation heat transfer in the system can, perhaps, lead to a desired product with a sought characteristics. Abd El-Aziz [18] studied the thermal-diffusion and diffusion-thermo effects on the heat and mass transfer characteristics of free convection past a continuously stretching permeable surface in the presence of magnetic field, blowing/suction, and radiation.

Osalusi et al. [19] investigated thermal-diffusion and diffusion-thermo effects on combined heat and mass transfer of a steady hydromagnetic convective and slip flow due to a rotating disk in the presence of viscous dissipation and Ohmic heating. Motsa [20] investigated the effect of both the Soret, and Dufour effects on the onset of double diffusive convection. Mansour et al. [21] investigated the effects of chemical reaction, thermal stratification, Soret number, and Dufour number on MHD-free convective heat and mass transfer of a viscous, incompressible, and electrically conducting fluid on a vertical stretching surface embedded in a saturated porous medium. Shateyi [22] investigated thermal radiation and buoyancy effects on heat and mass transfer over a semi-infinite stretching surface with suction and blowing.

Afify [23] carried out an analysis to study free convective heat and mass transfer of an incompressible, electrically conducting fluid over a stretching sheet in the presence of suction and injection with thermal-diffusion and diffusion-thermo effects. Hence, based on the mentioned investigations and applications, the present paper considers the effect of both the Soret, and Dufuor effects on MHD convective heat and mass transfer from vertical surfaces with Hall and radiation effects. Elgazery [24] analyzed numerically the problem of magneto-micropolar fluid flow, heat and mass transfer with suction and blowing through a porous medium under the effects of chemical reaction, Hall, ion-slip currents, variable viscosity, and variable thermal diffusivity.

Motivated by the above referenced work and the numerous possible industrial applications of the problem (like in isotope separation), it is of paramount interest in this study to investigate the effects of Hall currents, thermal radiation, Soret, and Dufour on boundary layer mixed convection MHD flow over a vertical surface in the presence of suction. None of the above investigations simultaneously studied the effects of Hall currents, thermal radiation, Soret, and Dufour on boundary layer mixed MHD flow over a vertical surface through a porous medium. Hence, the purpose of this paper is to extend Afify [23], to study the more general problem which includes thermal radiation, Soret, and Dufour effects on mixed convection MHD flow with heat and mass transfer past a vertical plate with suction through a porous medium in the presence of Hall currents.

The momentum, thermal, and solutal boundary layer equations are transformed into a set of ordinary differential equations and then solved using MATALAB bvp4c. The analysis of the results obtained in the present work shows that the flow field is appreciably influenced by Dufour and Soret numbers, Hall, and thermal radiation parameters and suction on the wall. To reveal the tendency of the solutions, selected results for the velocity components, temperature, and concentration are graphically depicted. The rest of the paper is structured as follows. In Section 2, we formulate the problem; in Section 3, we give the method of solution. 
Our results are presented and discussed in Section 4, and in Section 5, we present some brief conclusions.

\section{Mathematical Formulation}

We consider mixed free-forced convective and mass transfer flow of a viscous incompressible fluid over an isothermal semi-infinite vertical flat plate through a porous medium. The flow is steady, laminar, and three dimensional and the viscous fluid is an electrically conducting one. The flow configuration and coordinate system are as shown in Figure 1 with the $x$-axis along the vertical plate and the $y$-axis normal to it.

The $z$-direction coincides with the leading edge of the plate. A normal magnetic field is assumed to be applied in the $y$-direction and the induced magnetic field is negligible in comparison with the applied one which corresponds to very small magnetic Reynolds number. The surface is maintained at uniform constant temperature and concentration. The flow has significant thermal radiation, Hall, Soret, and Dufour effects. Under the electromagnetic Boussinesq approximations the basic boundary-layer equations are given by

$$
\begin{gathered}
\frac{\partial u}{\partial x}+\frac{\partial v}{\partial y}=0 \\
u \frac{\partial u}{\partial x}+v \frac{\partial u}{\partial y}=v \frac{\partial^{2} u}{\partial y^{2}}+g \beta_{t}\left(T-T_{\infty}\right)+g \beta_{c}\left(C-C_{\infty}\right)-\frac{\sigma B_{0}^{2}}{\rho\left(1+m^{2}\right)}(u+m w)-\frac{\mu}{\rho k^{*}} u \\
u \frac{\partial w}{\partial x}+v \frac{\partial w}{\partial y}=v \frac{\partial^{2} w}{\partial y^{2}}+\frac{\sigma B_{0}^{2}}{\rho\left(1+m^{2}\right)}(m u-w)-\frac{\mu}{\rho k^{*}} w \\
u \frac{\partial T}{\partial x}+v \frac{\partial T}{\partial y}=\frac{k}{\rho c_{p}} \frac{\partial^{2} T}{\partial y^{2}}+\frac{D k_{t}}{c_{s} C_{p}} \frac{\partial^{2} C}{\partial y^{2}}+\frac{\sigma B_{0}^{2}}{\rho c_{p}\left(1+m^{2}\right)}\left(u^{2}+w^{2}\right)-\frac{1}{\rho c_{p}} \frac{\partial q_{r}}{\partial y} \\
u \frac{\partial C}{\partial x}+v \frac{\partial C}{\partial y}=D \frac{\partial^{2} C}{\partial y^{2}}+\frac{D k_{t}}{T_{m}} \frac{\partial^{2} T}{\partial y^{2}}
\end{gathered}
$$

where $u, v$, and $w$ are the fluid velocity components along the $x-, y-$, and $z$-axes, respectively. $T$ and $C$ are the fluid temperature and concentration, respectively. $v$ is the kinematic viscosity, $\mu$ is the dynamic viscosity, $g$ is the gravitational force due to acceleration, $\rho$ is the density, $\beta_{t}$ is the coefficient of volume expansion, $\beta_{c}$ is the volumetric coefficient of expansion with concentration, $k$ is the thermal conductivity of the fluid, $B_{0}$ is the magnetic field of constant strength, $D$ is the coefficient of mass diffusivity, $c_{p}$ is the specific heat at constant pressure, $T_{m}$ is the mean fluid temperature, $k_{t}$ is the thermal diffusion ratio, $k^{*}$ is the permeability, $m$ is the Hall parameter, and $c_{S}$ is the concentration susceptibility.

The boundary conditions are

$$
\begin{gathered}
u(x, 0)=U_{s}=A_{0} x, \quad v(x, 0)=-V_{w}, \quad w(x, 0)=0, \quad T(x, 0)=T_{w}, \quad C(x, 0)=C_{w}, \\
u(x, \infty)=w(x, \infty)=0, \quad T(x, \infty)=T_{\infty}, \quad C(x, \infty)=C_{\infty} .
\end{gathered}
$$




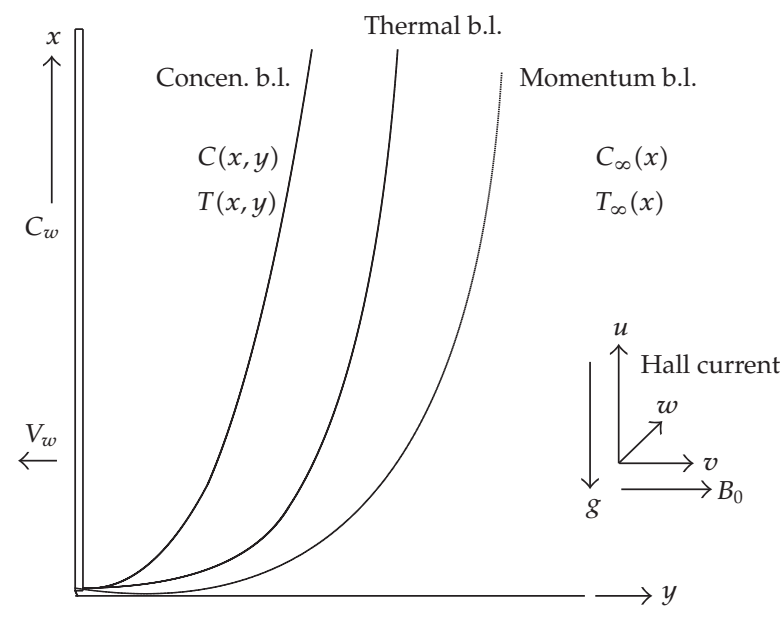

Figure 1: The coordinate system for the physical model of the problem.

$U_{s}$ is the surface velocity, $A_{0}$ is a constant with dimension (time $)^{-1}$, and $V_{w}, T_{w}$, and $C_{w}$ are the suction $(>0)$ or injection $(<0)$ velocity, the fluid temperature, and concentration at the plate, respectively. $T_{w}$ and $C_{w}$ are the temperature and concentration of the plate. The radiative heat flux $q_{r}$ is described by the Rosseland approximation such that

$$
q_{r}=-\frac{4 \sigma^{*}}{3 K} \frac{\partial T^{4}}{\partial y}
$$

where $\sigma^{*}$ and $K$ are the Stefan-Boltzman constant and the mean absorption coefficient, respectively. Following Chamkha [25], we assume that the temperature differences within the flow are sufficiently small so that the $T^{4}$ can be expressed as a linear function after using Taylor series to expand $T^{4}$ about the free stream temperature $T_{\infty}$ and neglecting higher-order terms. This results in the following approximation:

$$
T^{4} \approx 4 T_{\infty}^{3} T-3 T_{\infty}^{4}
$$

Using (2.7) and (2.8) in (2.4), we obtain

$$
\frac{\partial q_{r}}{\partial y}=-\frac{16 \sigma^{*} T_{\infty}^{3}}{3 K} \frac{\partial^{2} T}{\partial y^{2}}
$$

Following Elgazery [24] we nondimensionalize (2.1)-(2.5) using the following transformations:

$$
\begin{gathered}
\eta=\sqrt{\frac{A_{0}}{v}} y, \quad u=A_{0} x f^{\prime}(\eta), \quad v=-\sqrt{A_{0} v} f(\eta), \quad w=\sqrt{A_{0} v} h(\eta), \\
T=T_{\infty}+\left(T_{w}-T_{\infty}\right) \theta(\eta), \quad C=C_{\infty}+\left(C_{w}-C_{\infty}\right) \phi(\eta),
\end{gathered}
$$


where $f(\eta), h(\eta), \theta(\eta)$, and $\phi(\eta)$ are the dimensional stream, microrotation functions, temperature, and concentration distribution functions, respectively.

Upon substituting (2.10) into (2.1)-(2.5) we get the following similarity equations:

$$
\begin{gathered}
f^{\prime \prime \prime}+f f^{\prime \prime}-\left(f^{\prime}\right)^{2}+\operatorname{Gr} \theta+\operatorname{Gm} \phi-\frac{M}{1+m^{2}}\left(f^{\prime}+\frac{m}{\sqrt{\operatorname{Re}}} h\right)-\Omega f^{\prime}=0, \\
h^{\prime \prime}+f h^{\prime}+\frac{M}{1+m^{2}}\left(m \sqrt{\operatorname{Re}} f^{\prime}-h\right)-\Omega h=0, \\
\left(\frac{1}{\operatorname{Pr}}+R\right) \theta^{\prime \prime}+f \theta^{\prime}+\operatorname{Du} \phi^{\prime \prime}+\frac{M E c}{1+m^{2}}\left(\left(f^{\prime}\right)^{2}+\frac{h^{2}}{\operatorname{Re}}\right)=0, \\
\frac{1}{\mathrm{Sc}} \phi^{\prime \prime}+f \phi^{\prime}+\operatorname{Sr} \theta^{\prime \prime}=0,
\end{gathered}
$$

where the primes denote differentiation with respect to $\eta$. Where $M=\sigma B_{0}^{2} / \rho A_{0}$ is the magnetic parameter, $\operatorname{Pr}=v / \alpha$ is the Prandtl number, $\mathrm{Sc}=v / D$ is the Schmidt number, $\mathrm{Sr}=D k_{t}\left(T_{w}-T_{\infty}\right) / v T_{m}\left(C_{w}-C_{\infty}\right)$ is the Soret number, $\mathrm{Du}=D k_{t}\left(C_{w}-C_{\infty}\right) / v T_{m}\left(T_{w}-T_{\infty}\right)$ is the Dufour number, Gr $=g \beta_{t}\left(T_{w}-T_{\infty}\right) / U_{s} A_{0}$ is the local Grashof number, Gm = $g \beta_{c}\left(C_{w}-C_{\infty}\right) / U_{s} A_{0}$ is the local modified Grashof number, Ec $=U_{s}^{2} / c_{p}\left(T_{w}-T_{\infty}\right)$ is the Eckert number, $\Omega$ is the permeability parameter, $\operatorname{Re}=x U_{s} / v$ is the Reynolds number, $\mathrm{Sc}=v / D$ is the Schmidt number, and $R=4 \sigma T_{\infty}^{3} / k K$ is the dimensionless thermal radiation coefficient. In view of the similarity transformations, the boundary conditions transform into:

$$
\begin{gathered}
f(0)=f_{w}, \quad f^{\prime}(0)=1, \quad h(0)=0, \quad \theta(0)=1, \quad \phi(0)=1, \\
f^{\prime}(\infty)=0, \quad h(\infty)=0, \quad T(\infty)=0, \quad C(\infty)=0,
\end{gathered}
$$

where $f_{w}=V_{w} / \sqrt{A_{0} v}$ is the mass transfer coefficient such that $f_{w}>0$ indicates suction and $f_{w}<0$ indicates blowing at the surface.

\section{Method of Solution}

The governing nonlinear similarity equations (in the case $m=\mathrm{Gr}=\mathrm{Gm}=0$ ), together with the boundary conditions $f(0)=f_{w}, f^{\prime}(0)=1$, and $f^{\prime}(\infty)=0$, has an exact solution in the following form:

$$
f(\eta)=f_{w}+\frac{1}{a}\left(1-e^{-a \eta}\right), \quad a=\frac{f_{w}+\sqrt{f_{w}^{2}+4(M+\Omega+1)}}{2} .
$$

The full set of (2.11) were reduced to a system of first-order differential equations and solved using a MATLAB boundary value problem solver called bvp4c. This program solves boundary value problems for ordinary differential equations of the form $y^{\prime}=f(x, y, \mathbf{p}), a \leq$ $x \leq b$, by implementing a collocation method subject to general nonlinear, two-point boundary conditions $g(y(a), y(b), \mathbf{p})=0$. Here $\mathbf{p}$ is a vector of unknown parameters. Boundary value problems (BVPs) arise in most diverse forms. Just about any BVP can be 
Table 1: Values of the skin friction, $-f^{\prime \prime}(0)$, the exact solution, the present method, and results of Elgazery [24].

\begin{tabular}{lccc}
\hline$k_{p}$ & Exact solution & Present method & Elgazery [24] \\
\hline 1 & 1.417059704707229 & 1.417059704707229 & 1.4170597047066027 \\
2 & 1.269413474070165 & 1.269413474070165 & 1.2694134740920218 \\
5 & 1.173975065412817 & 1.173975065412817 & 1.1739750656338466 \\
10 & 1.140805151587558 & 1.140805151587558 & 1.1408051520832996 \\
15 & 1.129583274664413 & 1.129583274664413 & 1.1295832753169304 \\
\hline
\end{tabular}

formulated for solution with bvp4c. The first step is to write the $O D E s$ as a system of firstorder ordinary differential equations. The details of the solution method are presented in Shampine and Kierzenka [26] and references therein. The numerical results were compared with the exact solution for the skin friction $-f^{\prime \prime}(0)$ for various values of $k_{p}=1 / \Omega$. Table 1 gives a comparison between the exact solution for the skin friction, the present numerical scheme, and results obtained by Elgazery [24] who used a Chebyshev pseudospectral method of solution. The table shows an excellent agreement between our numerical results and the exact solution. The results in Table 1 were generated using $M=1, f_{w}=-0.7$, and $m=\mathrm{Gr}=$ $\mathrm{Gm}=0$

\section{Results and Discussion}

The similarity equations (2.11) are nonlinear, coupled ordinary differential equations which possess no closed-form solution. Thus, we solve these equations numerically subject to the boundary conditions given by (2.12). Graphical representations of the numerical results are illustrated in Figure 2 through Figure 23 to show the influences of different parameters on the boundary layer flow.

In this study, we investigate the influence of the Dufour and Soret effects separately in order to clearly observe their respective effects on the velocity, temperature, and concentration profiles of the flow. The variation of tangential velocity distribution with $\eta$ for different values of the Dufour variable Du is shown in Figure 2. It can be clearly seen in this figure that as the Dufour effects increase, the tangential velocity increases.

The variation of lateral velocity distribution with $\eta$ for different values of the Soret variable Sr is shown in Figure 3. It can be clearly seen that the velocity distribution in the boundary layer increases with the Soret parameter. In Figure 4 we observe that as the Eckert number value increases, the tangential velocity increases.

The effect of the magnetic field parameter $(M)$ is shown in Figure 5. It is observed that the tangential velocity of the fluid decreases with the increase of the magnetic field parameter values. The decrease in the tangential velocity as the Hartman number $(M)$ increases is because the presence of a magnetic field in an electrically conducting fluid introduces a force called the Lorentz force, which acts against the flow if the magnetic field is applied in the normal direction, as in the present study. This resistive force slows down the fluid velocity component as shown in Figure 5. Figure 6 depicts the tangential velocity profiles as the Hall parameter $m$ increases. We see that $f^{\prime}$ increases as $m$ increases. It can also be observed that $f^{\prime}$-profiles approach their classical values when the Hall parameter $m$ becomes large $(m>5)$. In Figure 7 we observe that the tangential velocity decreases as the values of the permeability parameter are increased as more fluid is taken away from the boundary layer. 


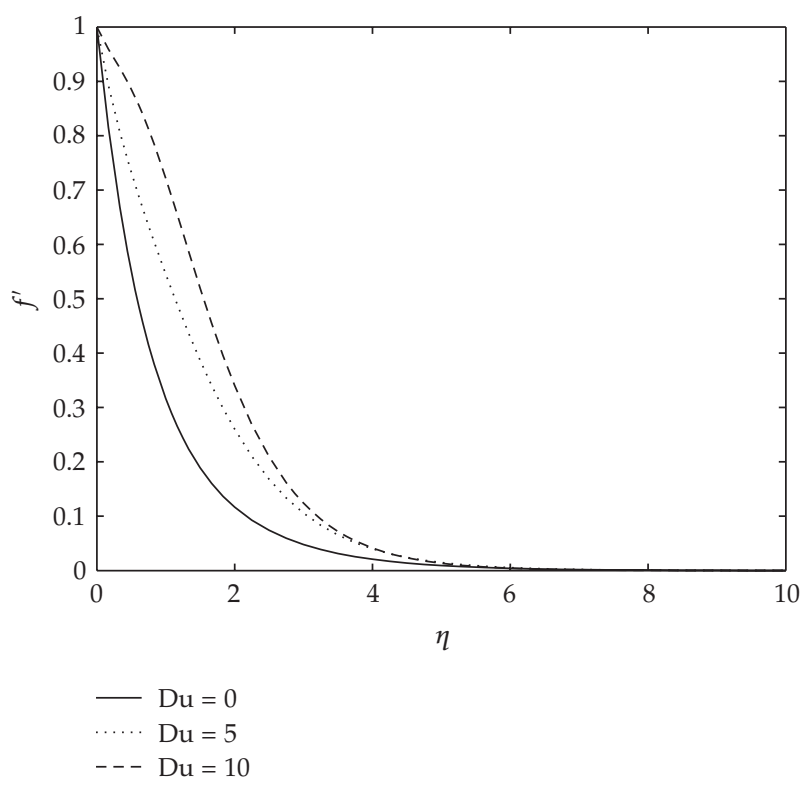

Figure 2: The variation of the tangential velocity distribution with increasing Dufour number with $A_{0}=$ $1, \mathrm{Gr}=\mathrm{Gm}=1, M=1, m=1, \operatorname{Pr}=0.72, R=1, \operatorname{Re}=1, \Omega=1, \mathrm{Sc}=1, \mathrm{Sr}=0$, and $\mathrm{Ec}=1$.

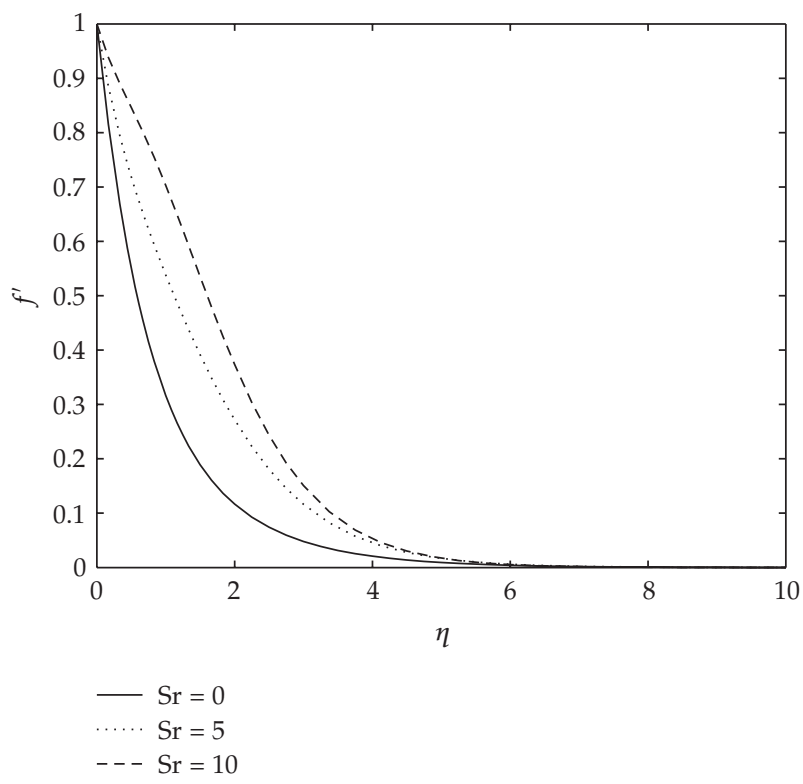

Figure 3: The variation of the tangential velocity distribution with increasing Dufour number with $A_{0}=$ $1, \mathrm{Gr}=\mathrm{Gm}=1, M=1, m=1, \operatorname{Pr}=0.72, R=1, \operatorname{Re}=1, \Omega=1, \mathrm{Sc}=1, \mathrm{Du}=0$, and $\mathrm{Ec}=1$.

The influence of thermal radiation on the tangential velocity is shown on Figure 8 . Increasing the thermal radiation parameter produces an increase in the tangential velocity of the flow. This is because large values of $R$ correspond to an increased dominance of conduction over absorption radiation thereby increasing buoyancy force and thickness of the momentum boundary layer (thus velocity). 


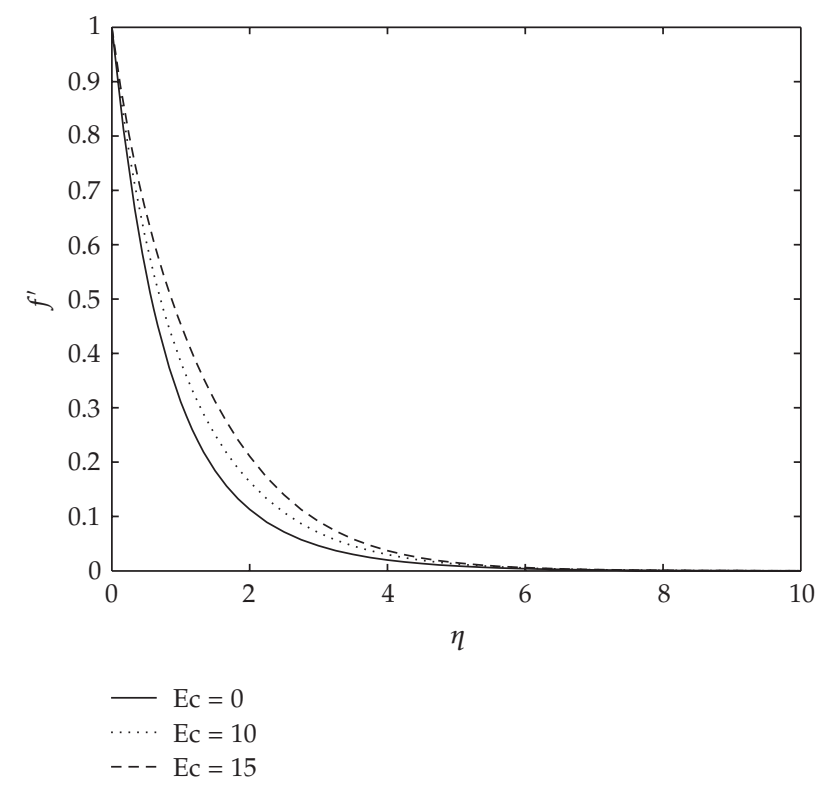

Figure 4: The variation of the tangential velocity distribution with increasing Eckert parameter with $A_{0}=$ $1, \mathrm{Gr}=\mathrm{Gm}=1, M=1, m=1, \operatorname{Pr}=0.72, R=1, \mathrm{Re}=1, \Omega=1, \mathrm{Sc}=1, \mathrm{Sr}=0$, and $\mathrm{Du}=0$.

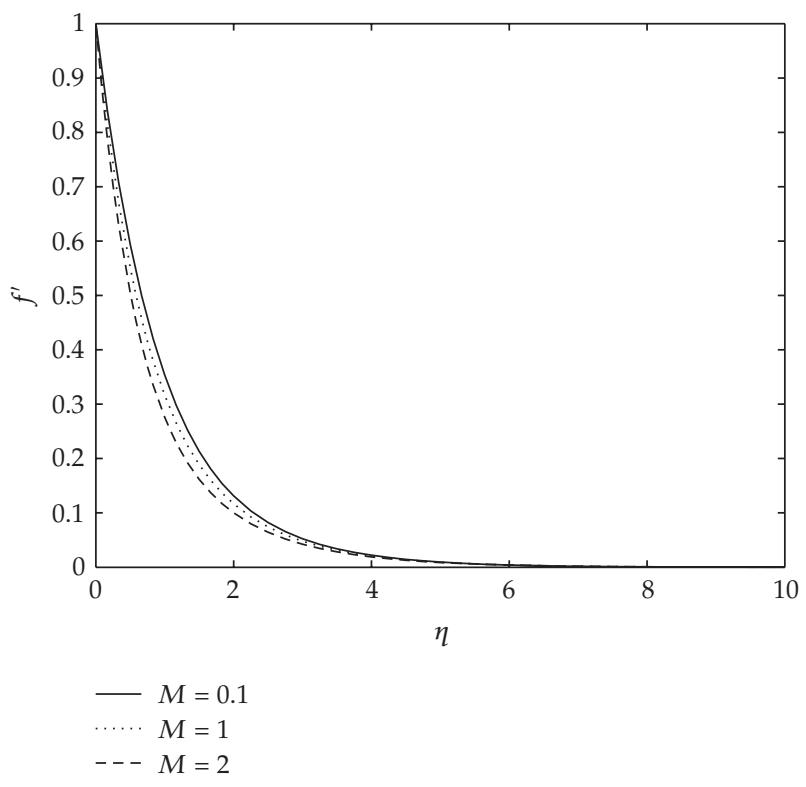

Figure 5: The variation of the tangential velocity distribution with increasing Hartman number parameter $A_{0}=1, \mathrm{Gr}=\mathrm{Gm}=1, m=1, \operatorname{Pr}=0.72, R=1, \operatorname{Re}=1, \Omega=1, \mathrm{Sc}=1, \mathrm{Sr}=0, \mathrm{Du}=0$, and $\mathrm{Ec}=1$.

The effects of Dufour parameter $(\mathrm{Du})$ are depicted in Figure 9. It is observed in this figure that the lateral velocity component increases with the increase in diffusion thermal effects. For each value of Dufour there exists a local maximum value for the lateral velocity 


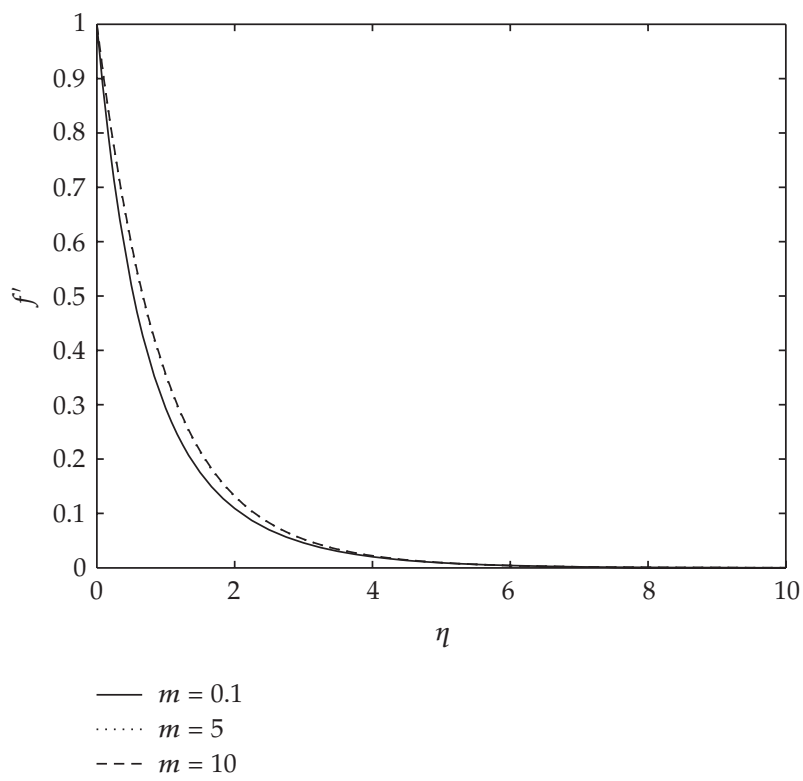

Figure 6: The variation of the tangential velocity distribution with increasing Hall parameter with $A_{0}=$ $1, \mathrm{Gr}=\mathrm{Gm}=1, M=1, \operatorname{Pr}=0.72, R=1, \operatorname{Re}=1, \Omega=1, \mathrm{Sc}=1, \mathrm{Sr}=0, \mathrm{Du}=0$, and $\mathrm{Ec}=1$.

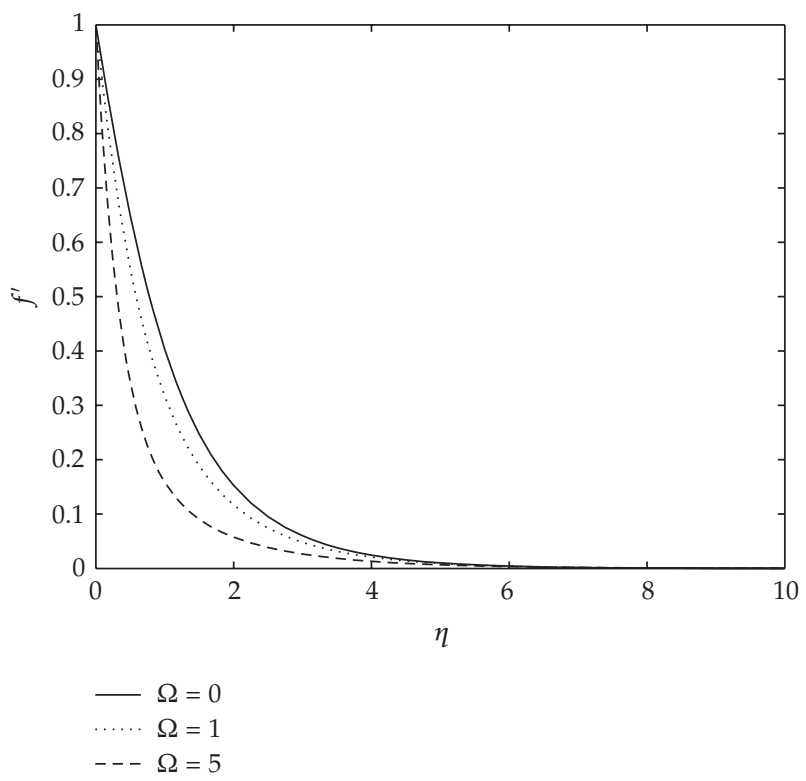

Figure 7: The variation of the tangential velocity distribution with increasing surface permeability parameter with $A_{0}=1, \mathrm{Gr}=\mathrm{Gm}=1, m=1, \operatorname{Pr}=0.72, R=1, \operatorname{Re}=1, M=1, \mathrm{Sc}=1, \mathrm{Sr}=0, \mathrm{Du}=$ 0 , and $\mathrm{Ec}=1$.

profile. Figure 10 shows the effect of Soret number on the lateral velocity distribution. From the figure it can be seen that this velocity component increases with the increase in Soret parameter Sr. It can also be seen that at each value of Sr there exist local maximum values in the lateral velocity profile in the boundary region. 


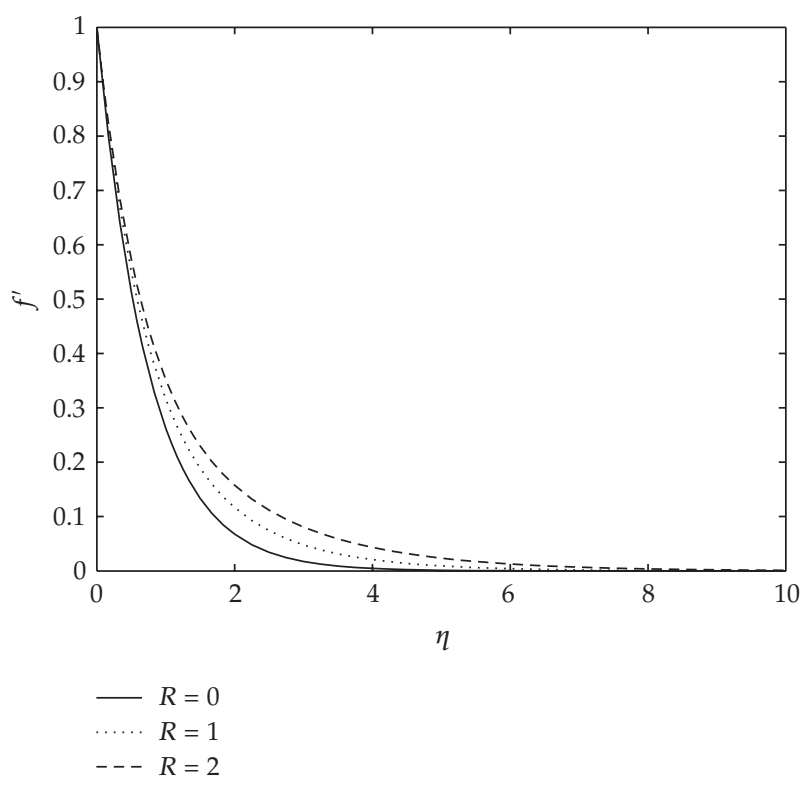

Figure 8: The variation of the tangential velocity distribution with increasing thermal radiation parameter with $A_{0}=1, \mathrm{Gr}=\mathrm{Gm}=1, m=1, \operatorname{Pr}=0.72, M=1, \operatorname{Re}=1, \Omega=1, \mathrm{Sc}=1, \mathrm{Sr}=0, \mathrm{Du}=0$, and $\mathrm{Ec}=1$.

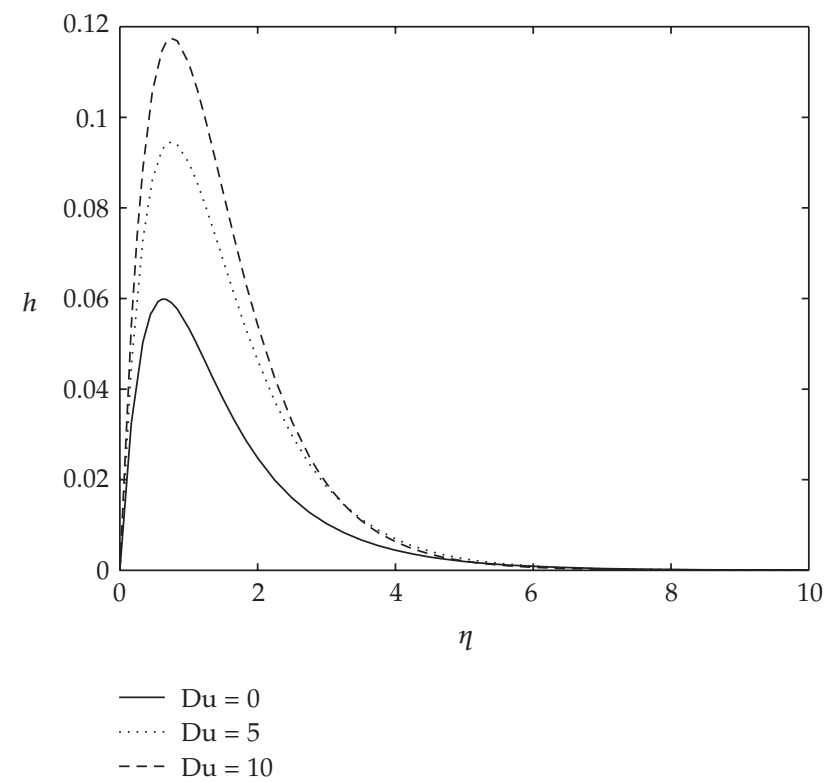

Figure 9: The variation of the lateral velocity distribution with increasing Dufour number with $A_{0}=$ 1, $\mathrm{Gr}=\mathrm{Gm}=1, M=1, m=1, \operatorname{Pr}=0.72, R=1, \operatorname{Re}=1, \Omega=1, \mathrm{Sc}=1, \mathrm{Sr}=0$, and $\mathrm{Ec}=1$.

In Figure 11 we have the influence of the magnetic field parameter on the lateral velocity. It can be seen that as the values of this parameter increase, the lateral velocity increases. 


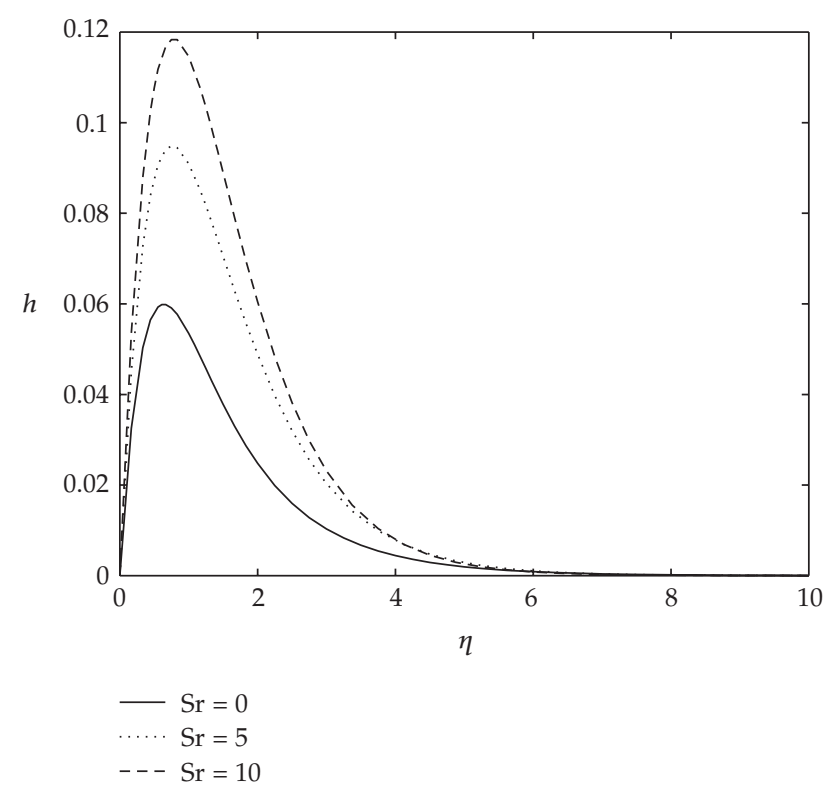

Figure 10: The variation of the lateral velocity distribution with increasing Soret number with $A_{0}=1, \mathrm{Gr}=$ $\mathrm{Gm}=1, M=1, m=1, \operatorname{Pr}=0.72, R=1, \operatorname{Re}=1, \Omega=1, \mathrm{Sc}=1, \mathrm{Du}=0$, and $\mathrm{Ec}=1$.

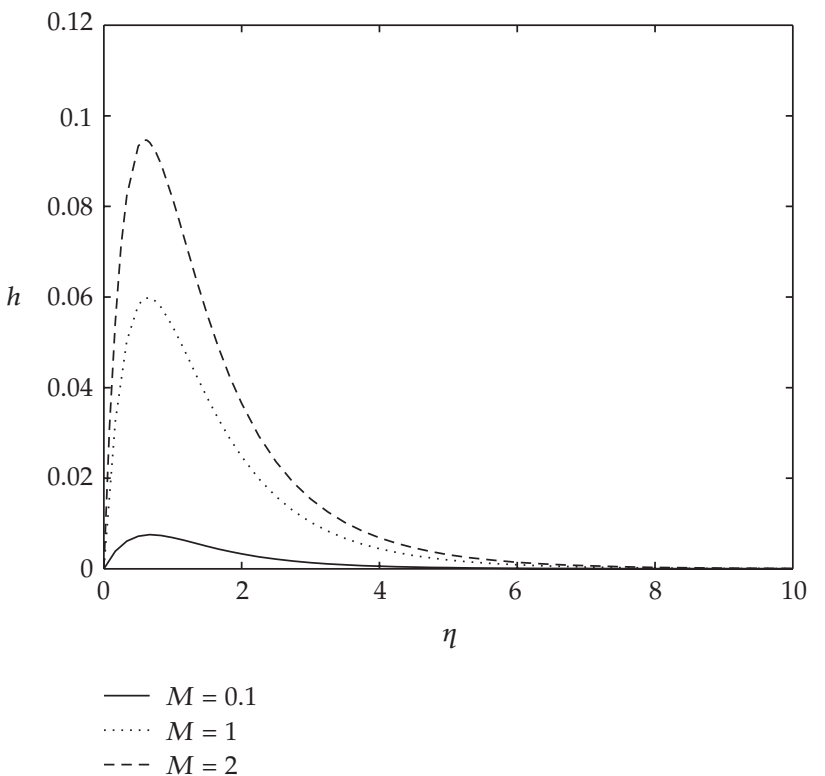

Figure 11: The variation of the lateral velocity distribution with increasing magnetic field with $A_{0}=1, \mathrm{Gr}=$ $\mathrm{Gm}=1, m=1, \operatorname{Pr}=0.72, R=1, \operatorname{Re}=1, \Omega=1, \mathrm{Sc}=1, \mathrm{Sr}=0, \mathrm{Du}=0$, and $\mathrm{Ec}=1$.

In Figure 12, we see that $h$-profiles increase for $m \leq 1$ and decrease for $m>1$. In Figure 13, we see the influence of the Eckert number on lateral velocity of the flow. It can be seen that as the Eckert number increases, this velocity component increases as well. Figure 14 shows that the increase of permeability of the surface reduces the lateral distribution of the 


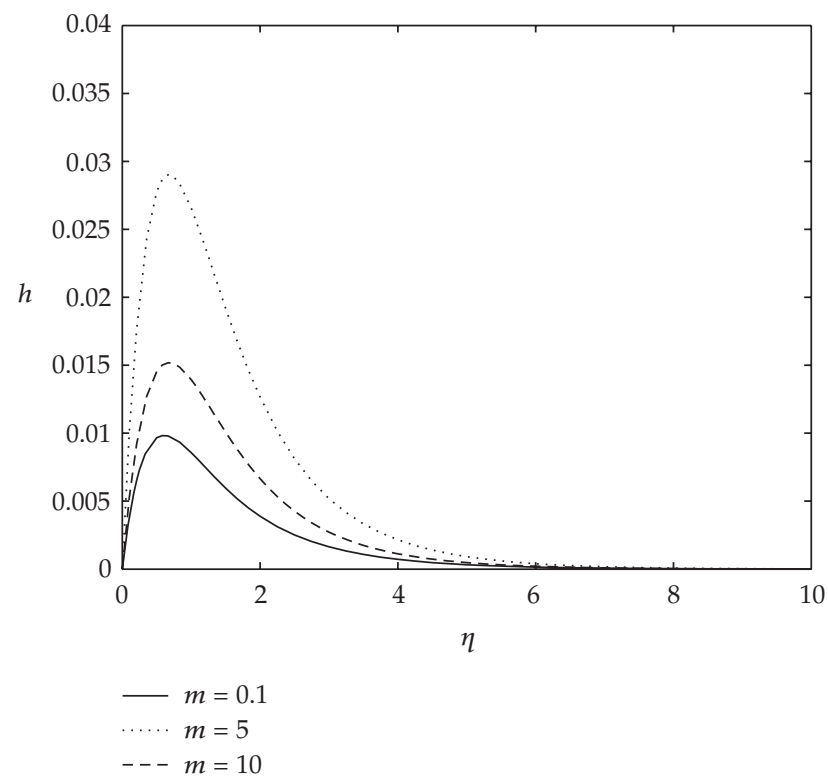

Figure 12: The variation of the lateral velocity distribution with increasing Hall current with $A_{0}=1, \mathrm{Gr}=$ $\mathrm{Gm}=1, M=1, \operatorname{Pr}=0.72, R=1, \operatorname{Re}=1, \Omega=1, \mathrm{Sc}=1, \mathrm{Sr}=0, \mathrm{Du}=0$, and $\mathrm{Ec}=1$.

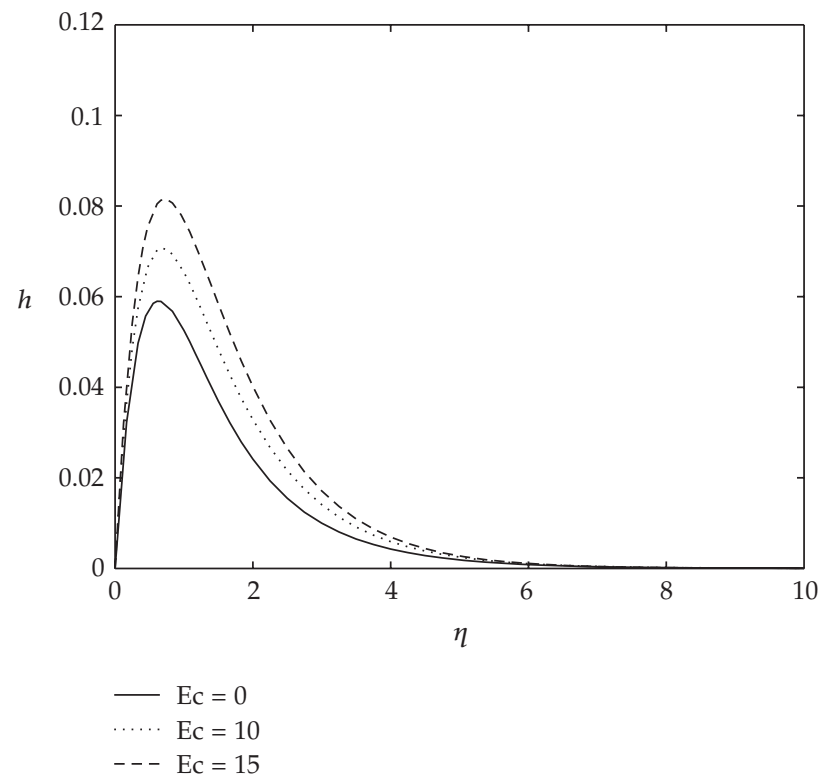

Figure 13: The variation of the lateral velocity distribution with increasing Eckert number with $A_{0}=$ $1, \mathrm{Gr}=\mathrm{Gm}=1, M=1, m=1, \operatorname{Pr}=0.72, R=1, \operatorname{Re}=1, \Omega=1, \mathrm{Sc}=1, \mathrm{Sr}=0$, and $\mathrm{Du}=0$.

fluid. The effects of thermal radiation on lateral velocity are shown in Figure 15. We observe that the lateral velocity increases as the value of the radiation parameter $R$ increases.

Figures 16 and 17 depict the behaviour of Du and Sr, on $\phi$, respectively. In Figure 16 we see the effects of the Dufour number on the concentration profiles. The Dufour effects reduce the concentration boundary layer in the fluid. 


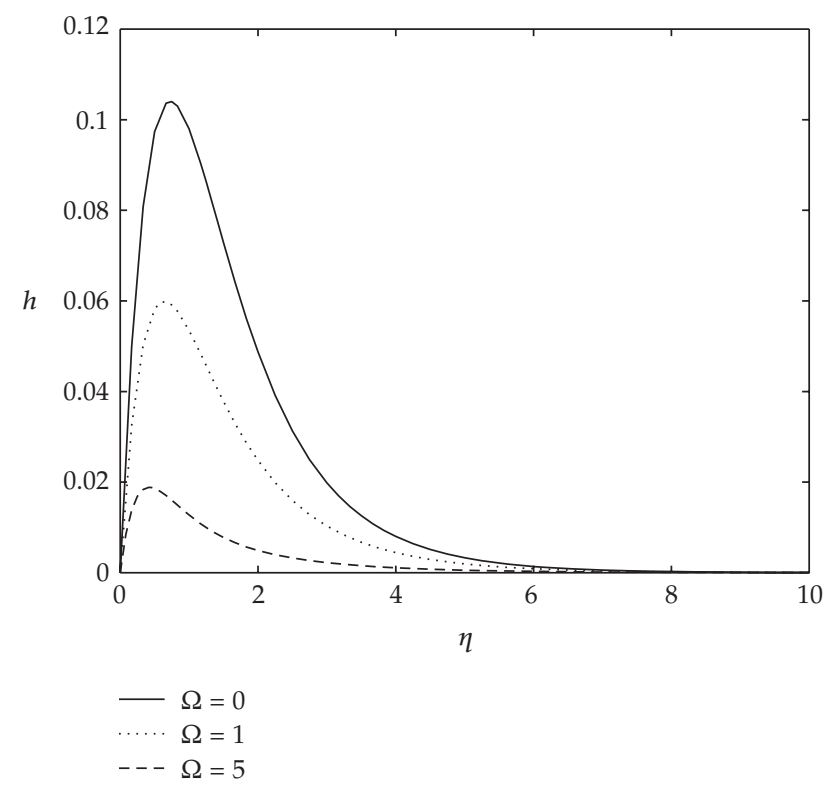

Figure 14: The variation of the lateral velocity distribution with increasing permeability number with $A_{0}=$ 1, $\mathrm{Gr}=\mathrm{Gm}=1, m=1, \operatorname{Pr}=0.72, R=1, \operatorname{Re}=1, M=1, \mathrm{Sc}=1, \mathrm{Sr}=0, \mathrm{Du}=0$, and $\mathrm{Ec}=1$.

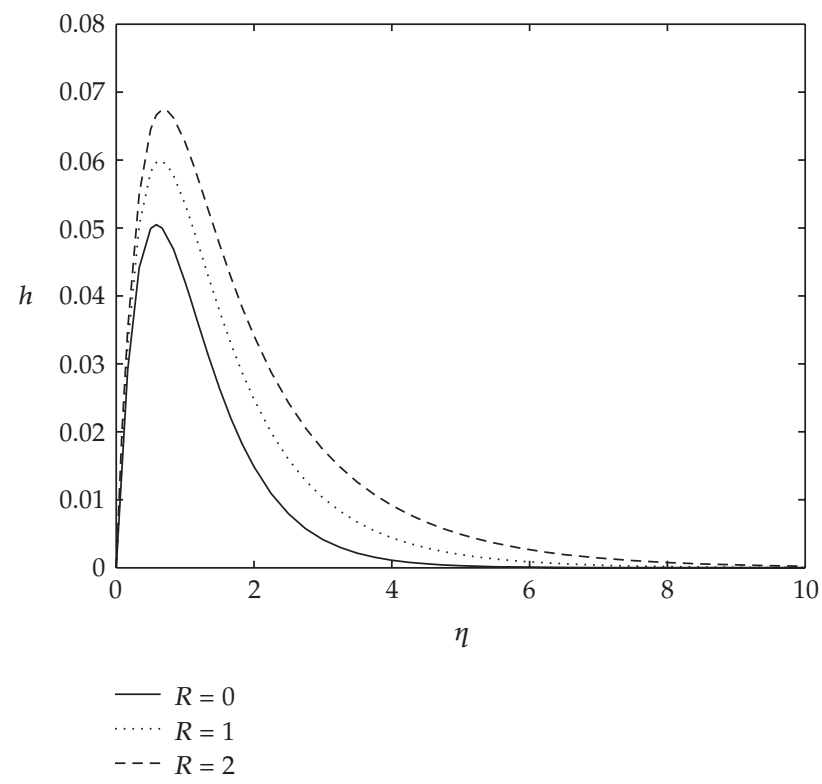

Figure 15: The variation of the lateral velocity distribution with increasing thermal radiation with $A_{0}=$ $1, \mathrm{Gr}=\mathrm{Gm}=1, m=1, \operatorname{Pr}=0.72, \operatorname{Re}=1, \Omega=0, M=1, \mathrm{Sc}=1, \mathrm{Sr}=0, \mathrm{Du}=0$, and $\mathrm{Ec}=1$.

Figure 17 shows the influence of the Soret parameter, Sr on the concentration profiles. It can be seen from this figure that the concentration $\phi(\eta)$ increases with increasing Sr values. From this figure we observe that the concentration profiles increase significantly with increase of the Soret number values. 


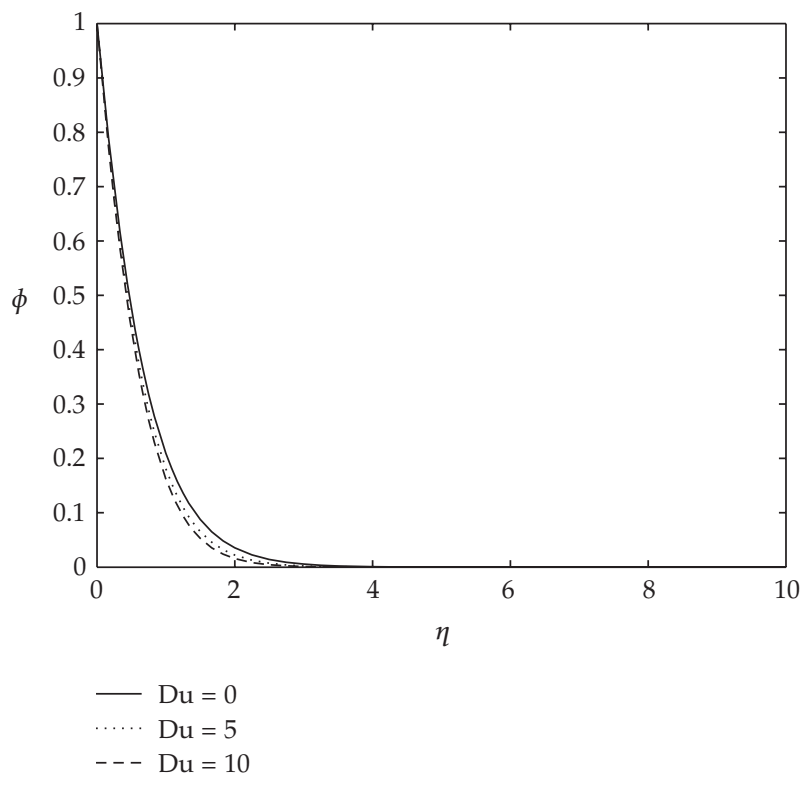

Figure 16: The variation of the concentration distribution with increasing Dufour number with $A_{0}=$ $1, \mathrm{Gr}=\mathrm{Gm}=1, m=1, \operatorname{Pr}=0.72, R=1, \operatorname{Re}=1, \Omega=1, M=1, \mathrm{Sr}=0$, and $\mathrm{Ec}=1$.

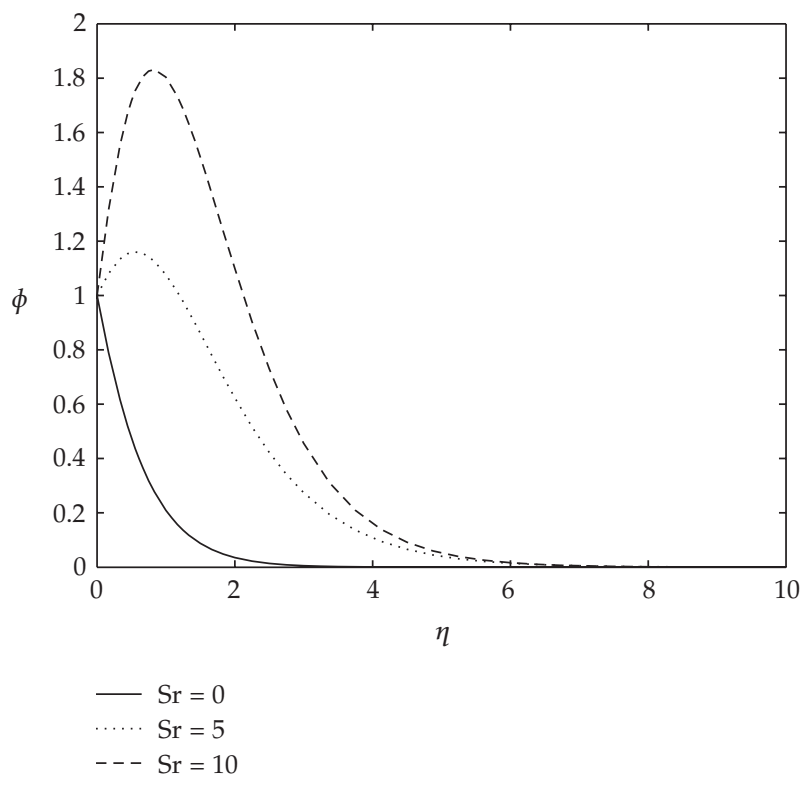

Figure 17: The variation of the concentration distribution with increasing Dufour number with $A_{0}=$ 1, $\mathrm{Gr}=\mathrm{Gm}=1, m=1, \operatorname{Pr}=0.72, R=1, \operatorname{Re}=1, \Omega=1, M=1, \mathrm{Du}=0$, and $\mathrm{Ec}=1$.

Figure 18 depicts the effects of the Dufour parameter on the fluid temperature. It can be clearly seen from this figure that diffusion thermal effects greatly affect the fluid temperature. As the values of the Dufour parameter increase, the fluid temperature also increases. 


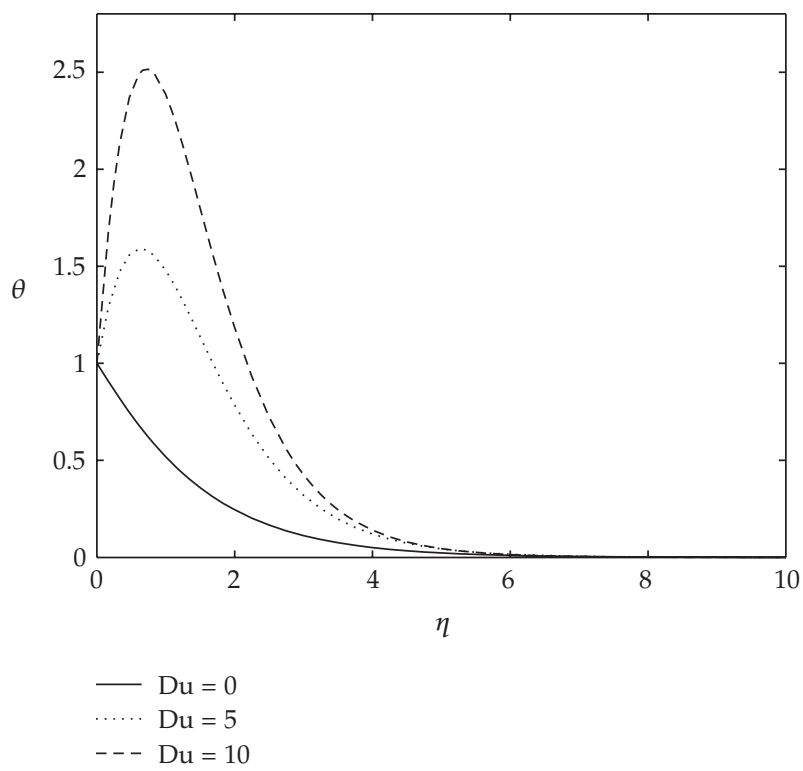

Figure 18: The variation of the temperature distribution with increasing Dufour number with $A_{0}=1, \mathrm{Gr}=$ $\mathrm{Gm}=1, m=1, \operatorname{Pr}=0.72, R=1, \operatorname{Re}=1, \Omega=1, M=1, \mathrm{Sr}=0$, and $\mathrm{Ec}=1$.

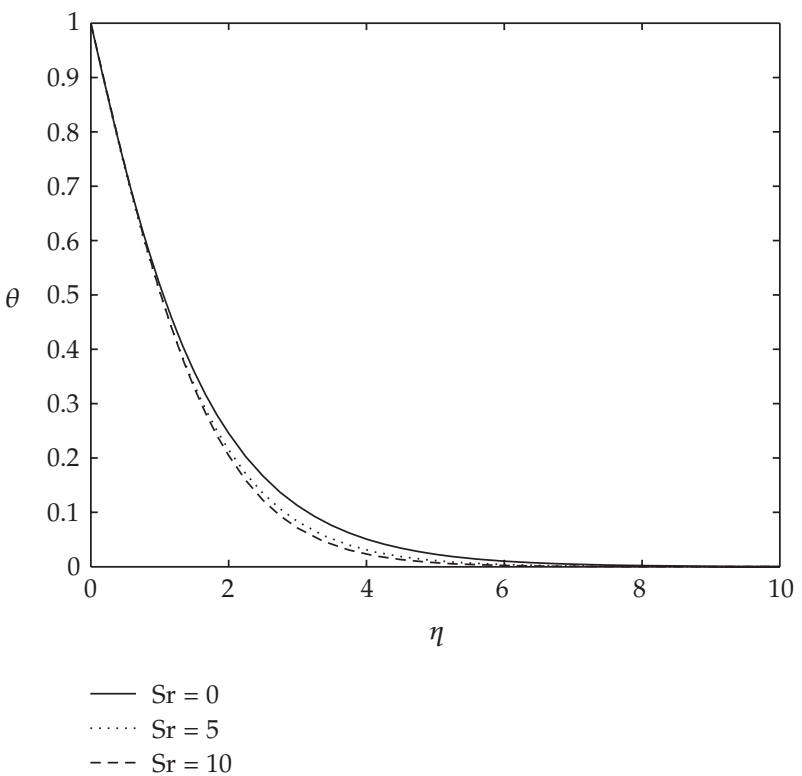

Figure 19: The variation of the temperature distribution with increasing Soret number with $A_{0}=1, \mathrm{Gr}=$ $\mathrm{Gm}=1, m=1, \operatorname{Pr}=0.72, R=1, \operatorname{Re}=1, \Omega=0, M=1, \mathrm{Du}=0$, and $\mathrm{Ec}=1$.

The influence of thermal-diffusion effects is shown in Figure 19. We observe that as $\mathrm{Sr}$ increases, there is a decrease in the temperature of the fluid though the changes are not very significant. As expected the effect of Soret number Sr on the temperature is quite opposite to that of $\mathrm{Du}$. It is also noticed that the behaviour of $\mathrm{Du}$ and $\mathrm{Sr}$ on the concentration and temperature distributions is opposite. 


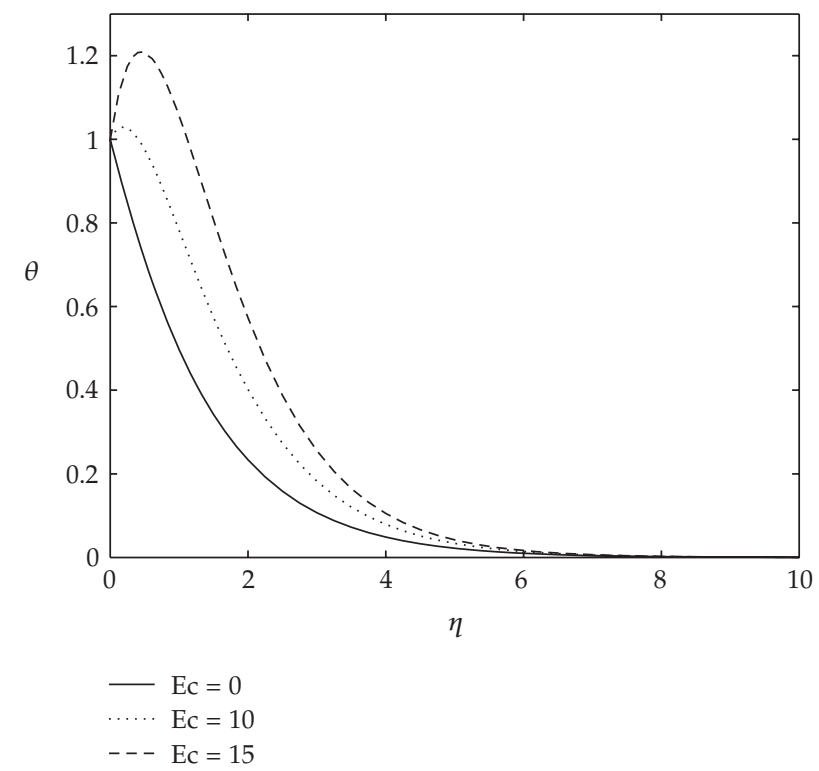

Figure 20: The variation of the concentration distribution with increasing Eckert number with $A_{0}=1, \mathrm{Gr}=$ $\mathrm{Gm}=1, m=1, \operatorname{Pr}=0.72, R=1, \operatorname{Re}=1, \Omega=1, M=1, \mathrm{Sc}=1, \mathrm{Sr}=0$, and $\mathrm{Du}=0$.

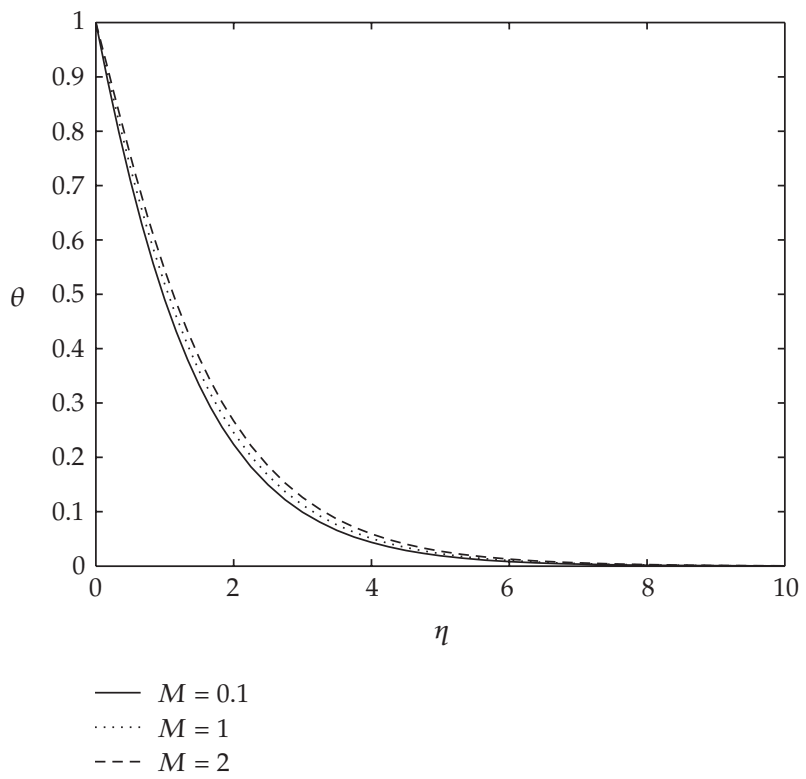

Figure 21: The variation of the temperature distribution with increasing magnetic field with $A_{0}=1, \mathrm{Gr}=$ $\mathrm{Gm}=1, m=1, \operatorname{Pr}=0.72, R=1, \operatorname{Re}=1, \Omega=1, \mathrm{Sc}=1, \mathrm{Sr}=0, \mathrm{Du}=0$, and $\mathrm{Ec}=1$.

In Figure 20 we see that the increase in the Eckert number values greatly affects the temperature of the fluid. The temperature of the fluid, increases as the Eckert number increases.

Figure 21 shows that the temperature boundary layer becomes thick by increasing the magnetic parameter. The effects of a transverse magnetic field give rise to a resistive-type 


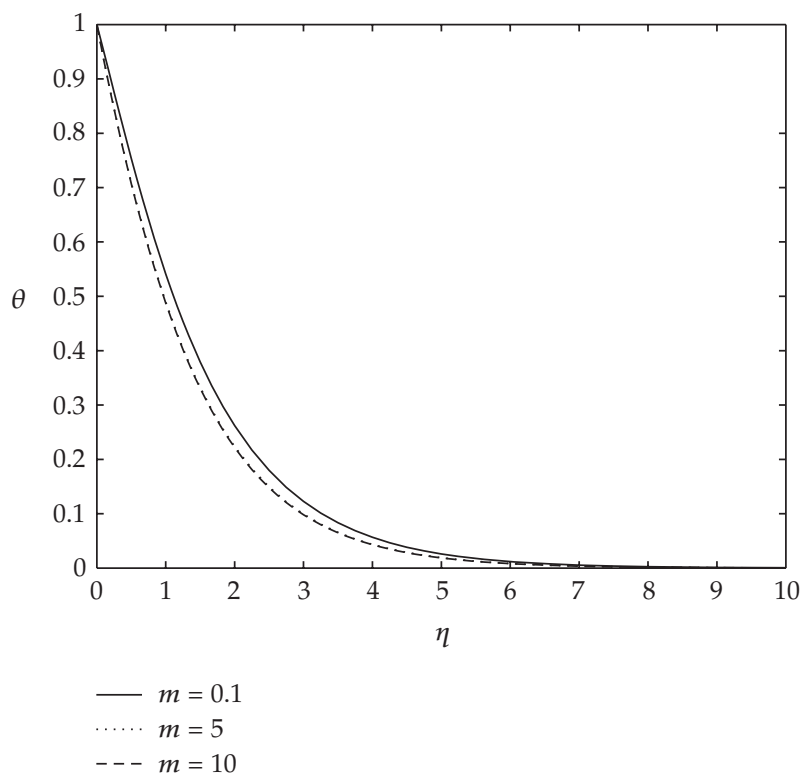

Figure 22: The variation of the temperature distribution with increasing Hall current with $A_{0}=1, \mathrm{Gr}=$ $\mathrm{Gm}=1, M=1, \operatorname{Pr}=0.72, R=1, \operatorname{Re}=1, \Omega=0, \mathrm{Sc}=1, \mathrm{Sr}=0, \mathrm{Du}=0$, and $\mathrm{Ec}=1$.

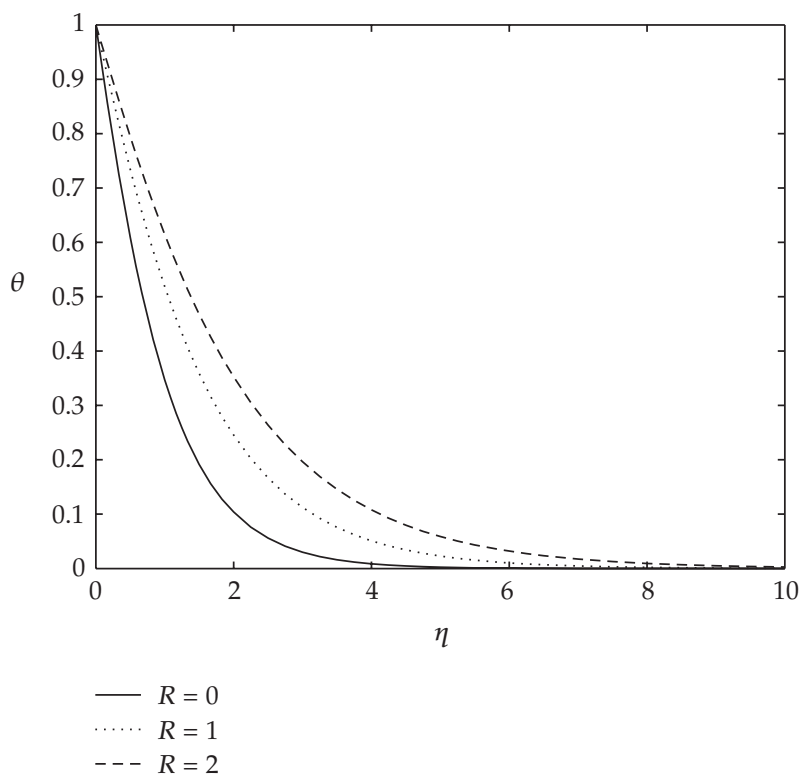

Figure 23: The variation of the temperature distribution with increasing thermal radiation with $A_{0}=$ 1, $\mathrm{Gr}=\mathrm{Gm}=1, m=1, \operatorname{Pr}=0.72, \Omega=1, \mathrm{Re}=1, \mathrm{Ec}=1, M=1, \mathrm{Sc}=1, \mathrm{Sr}=0$, and $\mathrm{Du}=0$.

force called the Lorentz force. This force has the tendency to slow down the motion of the fluid and to increase its thermal boundary layer hence increasing the temperature of the flow.

In Figure 22 we see that the temperature profiles approach their classical values when the Hall parameter $m$ becomes large. Temperature profiles $\theta$ decrease with increasing $m$. The effect of thermal radiation $R$ on the temperature profiles in the boundary layer is illustrated 
in Figure 23. It is obvious that an increase in the radiation parameter results in increasing the temperature profiles within the boundary layer as well as an increase in the thermal boundary layer thickness.

\section{Conclusion}

This work investigated the effects of diffusion-thermo and thermal-diffusion on MHD natural convection heat and mass transfer over a permeable vertical plate in the presence of radiation and hall current. The governing equations are approximated to a system of nonlinear ordinary differential equations by using suitable similarity transformations. Numerical calculations are carried out for various values of the dimensionless parameters of the problem using an efficient and easy to use MATLAB routine bvp4c. The results are presented graphically and we can conclude that the flow field and the quantities of physical interest are significantly influenced by these parameters. The velocity increases as the Soret, Dufour effects, Hall, Eckert parameter, and thermal radiation increase. However, the tangential velocity was found to decrease as the Hartman parameter increases and the lateral velocity component was increased by the increased values for the Hartman number. Both velocity components distributions were reduced by the increased values of the permeability of the plate.

The fluid temperature was found to increase as the Dufour parameter, Eckert number, magnetic strength, thermal radiation, and surface permeability increase and to decrease as the Hall current and Soret effects increase. The concentration decreases as the Dufour number, Eckert, Hall parameter, and radiation parameters increase and increases as the Soret effect, magnetic strength, and surface permeability increase.

In addition, the present analysis has shown that the Soret and Dufour numbers have significant effects on the distributions of the velocity, temperature, and concentration. We therefore conclude that thermal-diffusion and diffusion-thermo effects have to be considered in the fluid, heat, and mass transfer.

\section{References}

[1] Y. Jaluria, Natural Convection Heat and Mass Transfer, Pregamon Press, Oxford, UK, 1980.

[2] P. S. Gupta and A. S. Gupta, "Heat and mass transfer on a stretching sheet with suction or blowing," The Canadian Journal of Chemical Engineering, vol. 55, pp. 744-746, 1977.

[3] N. G. Kafoussias and E. W. Williams, "Thermal-diffusion and diffusion-thermo effects on mixed freeforced convective and mass transfer boundary layer flow with temperature dependent viscosity," International Journal of Engineering Science, vol. 33, no. 9, pp. 1369-1384, 1995.

[4] M. Kinyanjui, J. K. Kwanza, and S. M. Uppal, "Magnetohydrodynamic free convection heat and mass transfer of a heat generating fluid past an impulsively started infinite vertical porous plate with Hall current and radiation absorption," Energy Conversion and Management, vol. 42, no. 8, pp. 917-931, 2001.

[5] K. A. Yih, "The effect of transpiration on coupled heat and mass transfer in mixed convection over a vertical plate embedded in a saturated porous medium," International Communications in Heat and Mass Transfer, vol. 24, no. 2, pp. 265-275, 1997.

[6] E. M. A. Elbashbeshy, "The mixed convection along a vertical plate embedded in non-darcian porous medium with suction and injection," Applied Mathematics and Computation, vol. 136, no. 1, pp. 139-149, 2003.

[7] K. E. Chin, R. Nazar, N. M. Arifin, and I. Pop, “Effect of variable viscosity on mixed convection boundary layer flow over a vertical surface embedded in a porous medium," International Communications in Heat and Mass Transfer, vol. 34, no. 4, pp. 464-473, 2007.

[8] D. Pal and B. Talukdar, "Buoyancy and chemical reaction effects on MHD mixed convection heat and mass transfer in a porous medium with thermal radiation and Ohmic heating," Communications in Nonlinear Science and Numerical Simulation, vol. 15, no. 10, pp. 2878-2893, 2010. 
[9] S. Mukhopadhyay, "Effect of thermal radiation on unsteady mixed convection flow and heat transfer over a porous stretching surface in porous medium," International Journal of Heat and Mass Transfer, vol. 52, no. 13-14, pp. 3261-3265, 2009.

[10] T. Hayat, M. Mustafa, and I. Pop, "Heat and mass transfer for Soret and Dufour's effect on mixed convection boundary layer flow over a stretching vertical surface in a porous medium filled with a viscoelastic fluid," Communications in Nonlinear Science and Numerical Simulation, vol. 15, no. 5, pp. 1183-1196, 2010.

[11] A. Postelnicu, "Influence of a magnetic field on heat and mass transfer by natural convection from vertical surfaces in porous media considering Soret and Dufour effects," International Journal of Heat and Mass Transfer, vol. 47, no. 6-7, pp. 1467-1472, 2004.

[12] T. Lyubimova, E. Shyklyaeva, J. Legros, C. Shevtsova, and V. Roux, "Numerical study of high frequency vibration influence on measurement of Soret and diffusion coeffcients in low gravity conditions," Advances in Space Research, vol. 36, pp. 70-74, 2005.

[13] C. R. A. Abreu, M. F. Alfradique, and A. S. Telles, "Boundary layer flows with Dufour and Soret effects: i: forced and natural convection," Chemical Engineering Science, vol. 61, no. 13, pp. 4282-4289, 2006.

[14] S. Alam, M. M. Rahman, A. Maleque, and M. Ferdows, "Dufour and Soret effects on steady MHD combined free-forced convective and mass transfer flow past a semi-Infinite vertical plate," Thammasat International Journal of Science and Technology, vol. 11, no. 2, pp. 1-12, 2006.

[15] M. S. Alam and M. M. Rahman, "Dufour and Soret effects on mixed convection flow past a vertical porous flat plate with variable suction," Nonlinear Analysis: Modelling and Control, vol. 11, no. 1, pp. 3-12, 2006.

[16] M.-C. Li, Y.-W. Tian, and Y.-C. Zhai, "Soret and Dufour effects in strongly endothermic chemical reaction system of porous media," Transactions of Nonferrous Metals Society of China, vol. 16, no. 5, pp. 1200-1204, 2006.

[17] S. N. Gaikwad, M. S. Malashetty, and K. Rama Prasad, "An analytical study of linear and non-linear double diffusive convection with Soret and Dufour effects in couple stress fluid," International Journal of Non-Linear Mechanics, vol. 42, no. 7, pp. 903-913, 2007.

[18] M. Abd El-Aziz, "Thermal-diffusion and diffusion-thermo effects on combined heat and mass transfer by hydromagnetic three-dimensional free convection over a permeable stretching surface with radiation," Physics Letters, Section A, vol. 372, no. 3, pp. 263-272, 2008.

[19] E. Osalusi, J. Side, and R. Harris, "Thermal-diffusion and diffusion-thermo effects on combined heat and mass transfer of a steady MHD convective and slip flow due to a rotating disk with viscous dissipation and Ohmic heating," International Communications in Heat and Mass Transfer, vol. 35, no. 8, pp. 908-915, 2008.

[20] S. S. Motsa, "On the onset of convection in a porous layer in the presence of Dufour and Soret effects," SAMSA Journal of Pure and Applied Mathematics, vol. 3, pp. 58-65, 2008.

[21] M. A. Mansour, N. F. El-Anssary, and A. M. Aly, "Effects of chemical reaction and thermal stratification on MHD free convective heat and mass transfer over a vertical stretching surface embedded in a porous media considering Soret and Dufour numbers," Journal of Chemical Engineering, vol. 145, no. 2, pp. 340-345, 2008.

[22] S. Shateyi, "Thermal radiation and buoyancy effects on heat and mass transfer over a semi-infinite stretching surface with suction and blowing," Journal of Applied Mathematics, Article ID 414830, 12 pages, 2008.

[23] A. A. Afify, "Similarity solution in MHD: effects of thermal diffusion and diffusion thermo on free convective heat and mass transfer over a stretching surface considering suction or injection," Communications in Nonlinear Science and Numerical Simulation, vol. 14, no. 5, pp. 2202-2214, 2009.

[24] N. S. Elgazery, "The effects of chemical reaction, Hall and ion-slip currents on MHD flow with temperature dependent viscosity and thermal diffusivity," Communications in Nonlinear Science and Numerical Simulation, vol. 14, no. 4, pp. 1267-1283, 2009.

[25] A. J. Chamkha, "Hydromagnetic natural convetion from an isothermal inclined surface adjacent to a thermally stratified porous medium," International Journal of Engineering Science, vol. 37, no. 10-11, pp. 975-986, 1997.

[26] L. F. Shampine and J. Kierzenka, "Solving boundary value problems for ordinary differential equations in MATLAB with bvp4c," (Tutorial Notes), 2000. 


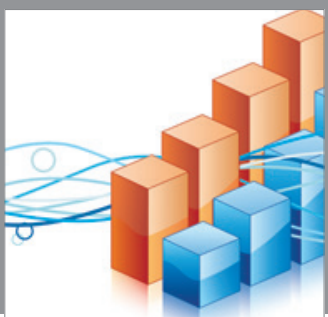

Advances in

Operations Research

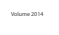

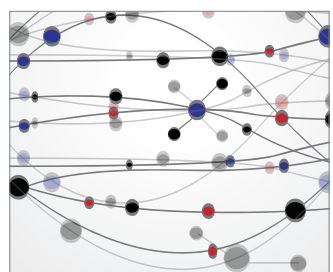

\section{The Scientific} World Journal
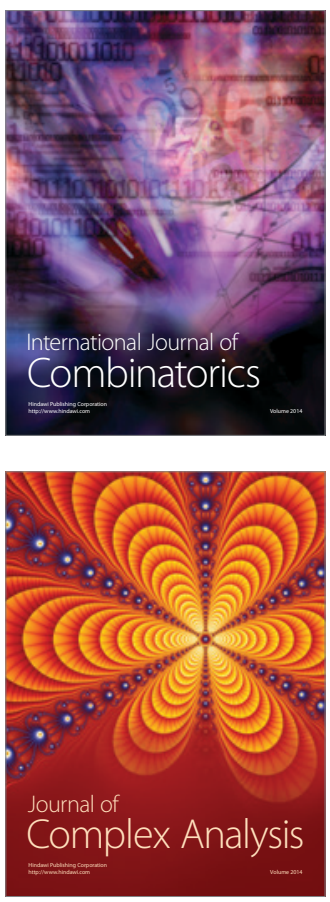

International Journal of

Mathematics and

Mathematical

Sciences
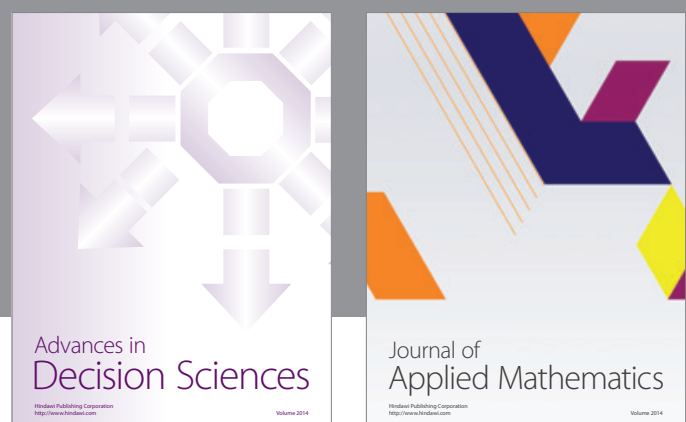

Journal of

Applied Mathematics
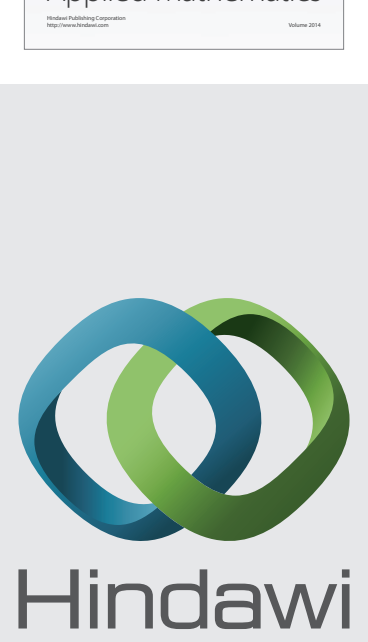

Submit your manuscripts at http://www.hindawi.com
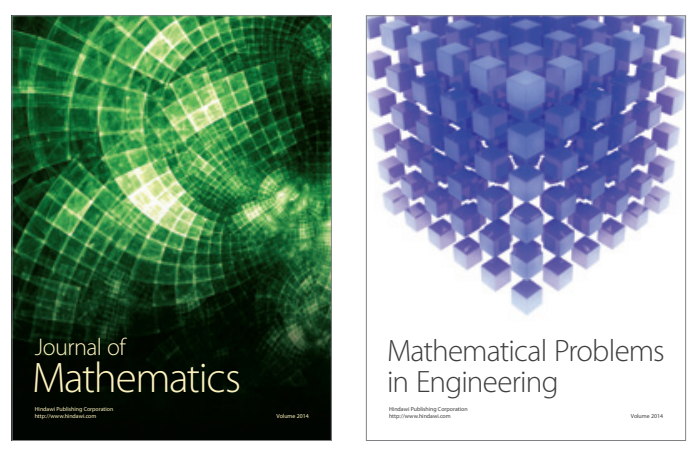

Mathematical Problems in Engineering
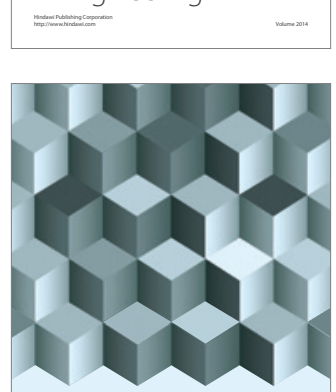

Journal of

Function Spaces
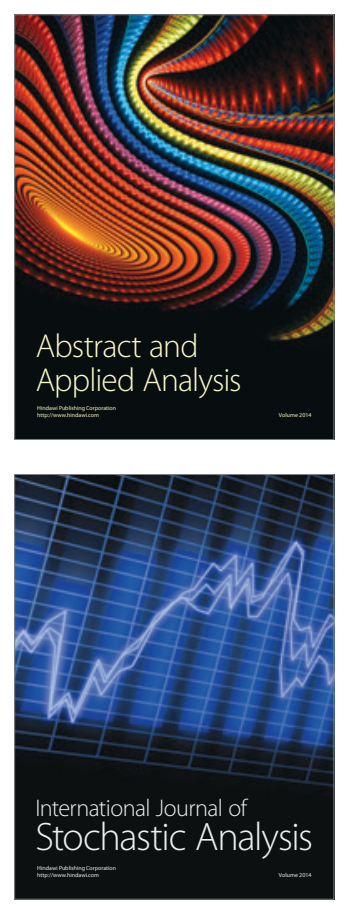

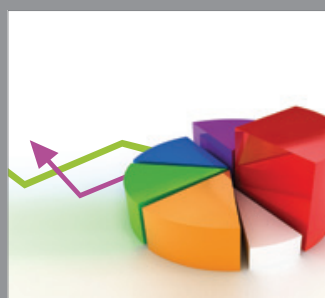

ournal of

Probability and Statistics

Promensencen
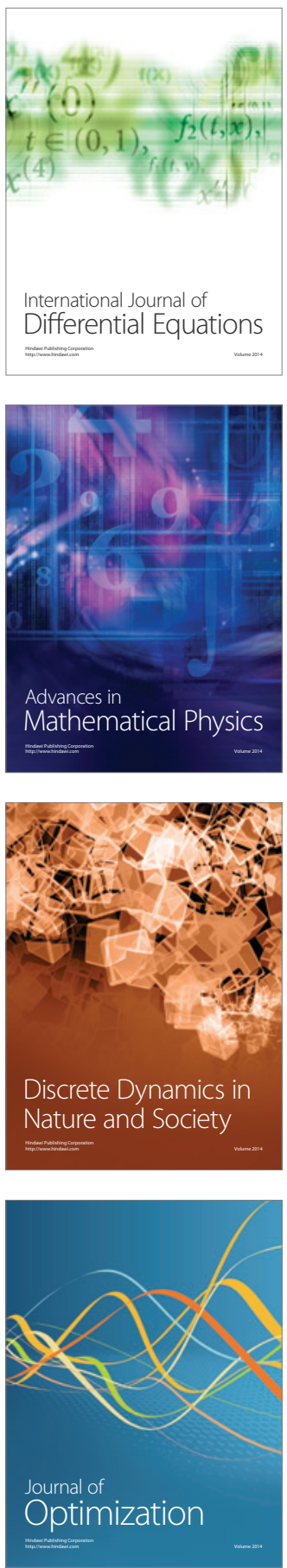